\title{
Implications and Limitations of Ideal Insulin Administration for People with Type 1
} Diabetes

Boiroux, Dimitri; Finan, Daniel Aaron; Jørgensen, John Bagterp; Poulsen, Niels Kjølstad; Madsen, Henrik

Published in:

UKACC International Conference on CONTROL 2010 proceedings

Publication date:

2010

Link back to DTU Orbit

Citation (APA):

Boiroux, D., Finan, D. A., Jørgensen, J. B., Poulsen, N. K., \& Madsen, H. (2010). Implications and Limitations of Ideal Insulin Administration for People with Type 1 Diabetes. In UKACC International Conference on CONTROL 2010 proceedings

\section{General rights}

Copyright and moral rights for the publications made accessible in the public portal are retained by the authors and/or other copyright owners and it is a condition of accessing publications that users recognise and abide by the legal requirements associated with these rights.

- Users may download and print one copy of any publication from the public portal for the purpose of private study or research.

- You may not further distribute the material or use it for any profit-making activity or commercial gain

- You may freely distribute the URL identifying the publication in the public portal

If you believe that this document breaches copyright please contact us providing details, and we will remove access to the work immediately and investigate your claim 


\title{
Implications and Limitations of Ideal Insulin Administration for People with Type 1 Diabetes *
}

\author{
Dimitri Boiroux* Daniel A. Finan* John Bagterp Jørgensen* \\ Niels Kjølstad Poulsen* Henrik Madsen* \\ * Department of Informatics and Mathematical Modelling, \\ Technical University of Denmark, DK-2800 Kgs Lyngby, Denmark \\ (e-mail: $\{$ dibo,dafi,jbj,nkp,hm\}@imm.dtu.dk)
}

\begin{abstract}
:
In this paper we use open-loop constrained non-linear optimal control to compute insulin administration profiles for people with type 1 diabetes. The algorithm is a multiple shooting algorithm based on sequential quadratic programming (SQP) for optimisation and an explicit Dormand-Prince Runge-Kutta method (DOPRI54) for numerical integration and sensitivity computation. We describe the numerical details of the constrained non-linear optimal control algorithm. The Hovorka model is used to describe a person with type 1 diabetes. We use the model and the algorithm to compute insulin administration profiles for people with type 1 diabetes in the cases with and without meal announcement in advance. The case with advance meal announcement results in almost perfect glucose control, but is undesirable as an insulin therapy due to the fact that most of the meal-related insulin is injected before the meal is actually taken. In the second, more realistic case, information about the meal is provided to the controller as the meal is taken. In this case, the optimal insulin administration profile is characterised by bolus-like injections of insulin coincident with the meals. These results indicate that, for certain conditions, insulin pens may be able to provide glucose control comparable to that of insulin pumps.
\end{abstract}

Keywords: type 1 diabetes, non-linear model predictive control, meal announcement

\section{INTRODUCTION}

The World Health Organization (2009) estimates that 180 million people worldwide have diabetes. This number is projected to double by 2030 . In the USA, the budget for diabetes represents approximately $10 \%$ of the health care budget, i.e., more than 130 billion dollars.

People with type 1 diabetes produce negligible amounts of pancreatic insulin. To maintain normal blood glucose concentrations, or normoglycaemia (approximately 60-140 $\mathrm{mg} / \mathrm{dL}$ or $3.3-7.8 \mathrm{mmol} / \mathrm{L}$ ), exogenous insulin must be injected. The glucose concentration must be regulated around $90 \mathrm{mg} / \mathrm{dL}$ and kept in the normoglycaemic range in order to avoid diabetes-related complications. Persistent high blood glucose concentrations above $140 \mathrm{mg} / \mathrm{dL}$ (hyperglycaemia) cause vascular, nerve, eye and kidney diseases. On the other hand, very low blood glucose concentrations (hypoglycaemia) can cause insulin shock or coma.

Insulin reduces the glucose concentration in the blood by facilitating the uptake of glucose into liver cells, where it can be stored as glycogen, and into peripheral tissue cells (muscles and adipose), where it can be stored or metabolised. Two types of insulin secretion patterns are

\footnotetext{
* Funding for this research as part of the DIACON project from the Danish Council for Strategic Research is gratefully acknowledged.
}

used by a normal, fully functional pancreas. First, a low, relatively constant basal rate of insulin is needed to counteract the glucose secreted by the conversion of glycogen to glucose in the liver (endogenous glucose production, or EGP). In addition to EGP, the major disturbance affecting the blood glucose levels is the absorption of carbohydrates (CHO) from meals. To offset these large loads of glucose, insulin is secreted in rapidly released boluses.

The challenge, then, for people with type 1 diabetes, is to try to mimic these insulin delivery patterns of a normal pancreas as closely as possible. Exogenous insulin therapy is often based on several (say, 4-8) blood glucose measurements per day, some of which are typically taken just before meals. Rapid-acting insulin analogues are injected as boluses to compensate for the $\mathrm{CHO}$ in the meal and correct the current blood glucose level if necessary. Longacting insulin analogues are taken infrequently (say, once per day) to counteract EGP. In well controlled subjects, the outcome of this therapy is illustrated in Fig. 1. Fig. 1 shows the daily glucose concentration tracings for one subject for seven consecutive days. Clearly, there is much room for improvement in the degree of glucose control. Significant parts of days are spent in the hyperglycaemic range, and occasionally hypoglycaemic events do occur.

Digestion and absorption of $\mathrm{CHO}$ from the gastrointestinal tract into the blood is generally faster than absorption of 


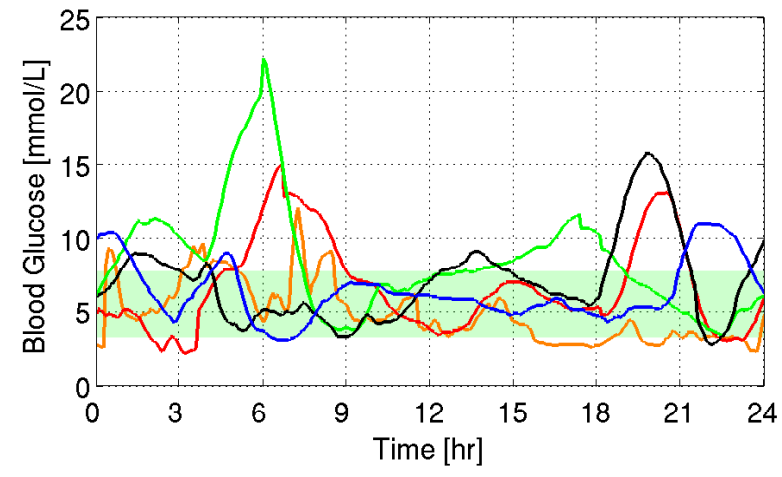

Fig. 1. Glucose concentration variations in a person with type 1 diabetes for 5 consecutive days using conventional insulin therapy based on discrete glucose measurements and discrete insulin injections. The greenshaded area represents the normoglycaemic range.

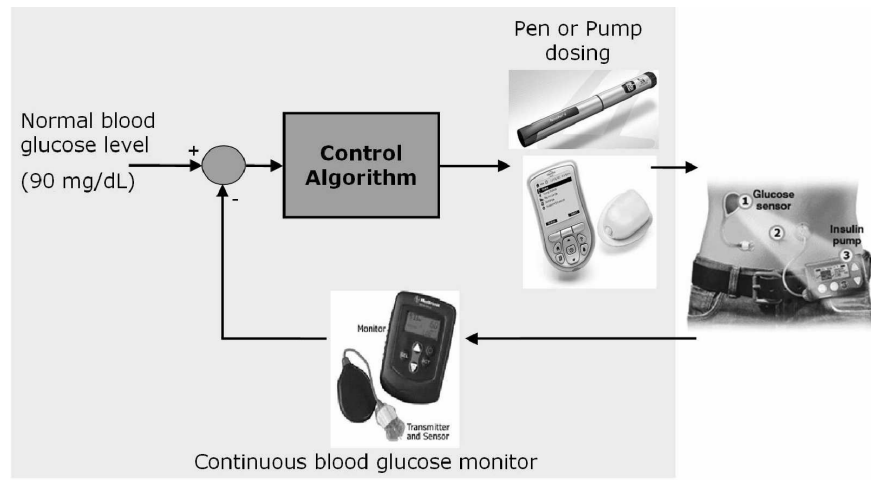

Fig. 2. Closed-loop glucose control. Glucose is measured subcutaneously using a continuous glucose monitor (CGM). Insulin is dosed either continuously by an insulin pump or discretely using an insulin pen.

subcutaneously injected insulin into the blood. Furthermore, the glucose-insulin dynamics are complex and nonlinear. Thus, with infrequent, discrete measurements of blood glucose and estimates of the CHO content in meals, it is not surprising that the degree of control depicted in Fig. 1 is typical of conventional insulin therapy.

To improve the glycaemic control by fine-tuning their insulin therapy, people with type 1 diabetes are using continuous glucose monitors (CGMs) more prevalently. These CGMs continuously (every one to few minutes) measure the subcutaneous glucose concentration. In addition, insulin pumps that can continuously infuse rapid-acting insulin are becoming more popular. The combination of these two medical devices has inspired much research interest in an artificial beta-cell (pancreas) that automatically adjusts the insulin dosage to control the blood glucose in people with type 1 diabetes. This concept is illustrated in Fig. 2. Several research groups worldwide are investigating aspects of control algorithms integrating the CGM and the insulin pump to automatically adjust insulin administration for people with type 1 diabetes, such as Klonoff et al. (2009).

The quality of the glucose control is limited by the time lag associated with subcutaneous-to-intravenous insulin absorption. In some mathematical descriptions of the phys-

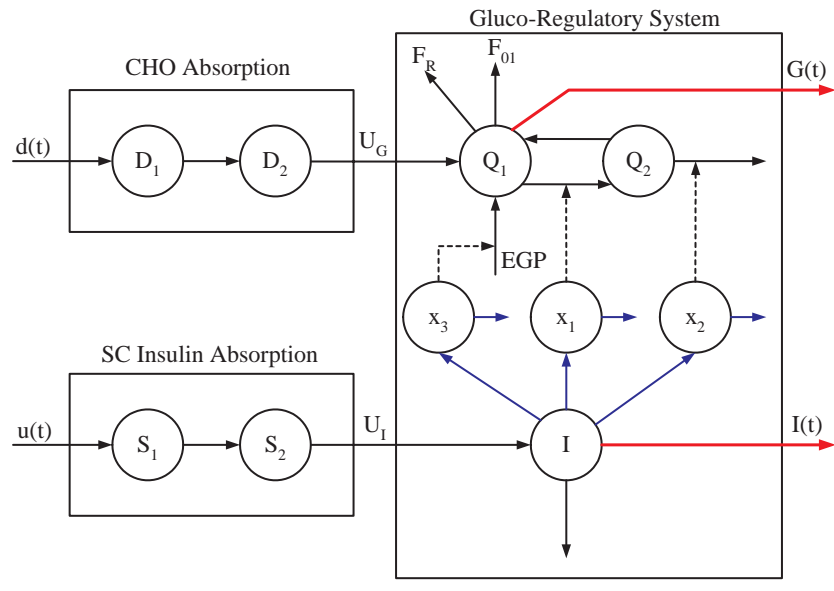

Fig. 3. Diagram of the Hovorka model.

iology, such as that of Hovorka et al. (2004), the absorption and transport of subcutaneously injected insulin to systemic circulation is modeled as linear second order process with time constants of $\tau_{S}=55 \mathrm{~min}$. Digestion and absorption of CHO is similarly modelled as a linear second order process, but with time constants of $\tau_{D}=40 \mathrm{~min}$. These absorption models and their relation to glucoseinsulin dynamics in the Hovorka model are illustrated in Fig. 3. This system property fundamentally limits the control quality that can be achieved in closed-loop insulin administration.

In this paper, we use constrained non-linear optimal control theory to compute the optimal insulin injection rates during a day. The computed profiles are open loop profiles and are not based on feedback. We consider the case when the meals throughout the day are announced to the controller in advance, and the case when the meals are only announced to the controller when they are taken. Knowing the ideal insulin administration profiles, we compare and discuss the advantages, disadvantages, and implications of these solutions. In particular, we address the issue of whether we can expect fundamentally better insulin therapy with pumps than with pens in the limiting case of perfect meal information.

The paper is structured as follows. The constrained nonlinear optimal control problem is presented in Section 2. Section 3 presents an quasi-Newton SQP algorithm with line search for solution of the discrete-time constrained non-linear Bolza problem. The numerical procedures for integration of the differential equations and computation of their sensitivities are also presented in Section 3. The non-linear model predictive controller and the scenarios are stated in Section 4. Section 5 applies the constrained non-linear optimal control algorithm to compute insulin administration profiles for virtual subjects with type 1 diabetes. Conclusions are provided in Section 6 .

\section{PROBLEM FORMULATION}

In this section, we state and discuss the continuous-time optimal control problem that we use to compute the insulin injection profiles for people with type 1 diabetes. We also discuss a numerically tractable discrete-time approximation to the continuous-time optimal control problem. 
The bound constrained continuous-time Bolza problem

$$
\begin{array}{ll}
\min _{[x(t), u(t)]_{t_{0}}^{t_{f}}} & \phi=\int_{t_{0}}^{t_{f}} g(x(t), u(t)) d t+h\left(x\left(t_{f}\right)\right) \\
\text { s.t. } & x\left(t_{0}\right)=x_{0} \\
& \dot{x}(t)=f(x(t), u(t), d(t)) \\
& u_{\min } \leq u(t) \leq u_{\max }
\end{array}
$$

is used to compute the optimal insulin administration. $x(t) \in \mathbf{R}^{n_{x}}$ is the state vector, $u(t) \in \mathbf{R}^{n_{u}}$ is the manipulated inputs, and $d(t) \in \mathbf{R}^{n_{d}}$ are known disturbances. $\dot{x}(t)=f(x(t), u(t), d(t))$ represents the model equations. The initial time, $t_{0}$, and the final time, $t_{f}$, are specified parameters. The initial state, $x_{0}$, is a known parameter in (1). The inputs are bound constrained and must be in the interval $u(t) \in\left[u_{\min }, u_{\max }\right]$.

The objective function is stated generally with a stage cost term, $g(x(t), u(t))$, and a cost-to-go term, $h\left(x\left(t_{f}\right)\right)$. The numerical algorithms for the problem are derived using this general structure of the objective function.

In the insulin administration problem, the stage cost term is a penalty function, the cost-to-go term is zero, and the model equations are represented by the Hovorka model Hovorka et al. (2004). $u(t)$ represents the rate of insulin injection at any time and $d(t)$ represents the carbohydrates $(\mathrm{CHO})$ intake rate at any time. Given an initial state, $x_{0}$, and a CHO intake rate profile, $[d(t)]_{t_{0}}^{t_{f}}$, the continuous-time Bolza problem (1) computes the optimal insulin injection rate profile, $[u(t)]_{t_{0}}^{t_{f}}$, as well as the optimal state trajectory, $[x(t)]_{t_{0}}^{t_{f}}$.

\subsection{Discrete-Time Approximation}

The continuous-time bound constrained Bolza problem (1) is approximated by a numerical tractable discrete-time bound constrained Bolza problem using the zero-orderhold input parametrisation of the manipulated variables, $u(t)$, as well as the known disturbance variables, $d(t)$. We divide the time interval, $\left[t_{0}, t_{f}\right]$, into $N$ equidistant intervals each of length $T_{s}$. Let $\mathcal{N}=\{0,1, \ldots, N-1\}$ and $t_{k}=t_{0}+k T_{s}$ for $k \in \mathcal{N}$. The zero-order-hold restriction on the input variables, $u(t)$ and $d(t)$, imply

$$
\begin{aligned}
& u(t)=u_{k} \quad t_{k} \leq t<t_{k+1} \quad k \in \mathcal{N} \\
& d(t)=d_{k} \quad t_{k} \leq t<t_{k+1} \quad k \in \mathcal{N}
\end{aligned}
$$

Using this zero-order-hold restriction on the inputs, the bound constrained continuous-time Bolza problem may be expressed as

$$
\begin{array}{lll}
\min _{\left\{x_{k+1}, u_{k}\right\}_{k=0}^{N-1}} & \phi=\sum_{k=0}^{N-1} G_{k}\left(x_{k}, u_{k}, d_{k}\right)+h\left(x_{N}\right) \\
\text { s.t. } & b_{k}:=F_{k}\left(x_{k}, u_{k}, d_{k}\right)-x_{k+1}=0 & k \in \mathcal{N} \\
& u_{\min } \leq u_{k} \leq u_{\max } & k \in \mathcal{N}
\end{array}
$$

The discrete-time state transition function is and the discrete time stage cost is

$$
\begin{aligned}
G_{k}\left(x_{k}, u_{k}, d_{k}\right)=\left\{\int_{t_{k}}^{t_{k+1}}\right. & g\left(x(t), u_{k}\right) d t \\
\dot{x}(t) & \left.=f\left(x(t), u_{k}, d_{k}\right), x\left(t_{k}\right)=x_{k}\right\}
\end{aligned}
$$

\section{NUMERICAL OPTIMISATION ALGORITHM}

In this section, we develop a multiple-shooting based SQP algorithm described in Diehl et al. (2009) and in Bock and Plitt (1984) for the numerical solution of (1). The SQP algorithm is based on line search. The structure of the quadratic sub-problems are utilised and they are solved by a primal-dual interior-point algorithm using Riccati iterations, as in Jørgensen (2005) and Rao et al. (1998). The DOPRI54 scheme derived in Dormand and Prince (1980) is used for numerical solution of the differential equation model and sensitivities.

\subsection{SQP Algorithm}

Define the parameter vector, $p$, as

$$
p=\left[\begin{array}{llllllll}
u_{0}^{\prime} & x_{1}^{\prime} & u_{1}^{\prime} & x_{2}^{\prime} & \ldots & x_{N-1}^{\prime} & u_{N-1}^{\prime} & x_{N}^{\prime}
\end{array}\right]^{\prime},
$$

and the disturbance vector, $d=\left[\begin{array}{llll}d_{0}^{\prime} & d_{1}^{\prime} & \ldots & d_{N-1}^{\prime}\end{array}\right]^{\prime}$, such that the discrete time dynamics may be represented by

$$
b(p)=b\left(p ; x_{0}, d\right)=\left[\begin{array}{c}
F_{0}\left(x_{0}, u_{0}, d_{0}\right)-x_{1} \\
F_{1}\left(x_{1}, u_{1}, d_{1}\right)-x_{2} \\
\vdots \\
F_{N-1}\left(x_{N-1}, u_{N-1}, d_{N-1}\right)-x_{N}
\end{array}\right]
$$

and the objective function may be denoted

$$
\phi(p)=\phi\left(p ; x_{0}, d\right)=\sum_{k=0}^{N-1} G_{k}\left(x_{k}, u_{k}, d\right)+h\left(x_{N}\right)
$$

Let $c(p)$ denote the bound constraints, i.e.

$$
c(p)=\left[\begin{array}{c}
u_{0}-u_{\min } \\
u_{1}-u_{\min } \\
\vdots \\
u_{N-1}-u_{\min } \\
u_{\max }-u_{0} \\
u_{\max }-u_{1} \\
\vdots \\
u_{\max }-u_{N-1}
\end{array}\right] .
$$

Then the bound constrained discrete-time Bolza problem may be expressed as a constrained optimisation problem in standard form

$$
\begin{array}{cl}
\min _{p} & \phi=\phi(p) \\
\text { s.t. } & b(p)=0 \\
& c(p) \geq 0
\end{array}
$$

The concise formulation (10) is useful for presentation of the numerical optimisation algorithm used for solving the bound constrained continuous-time Bolza problem (1).

$F_{k}\left(x_{k}, u_{k}, d_{k}\right)=\left\{x\left(t_{k+1}\right): \dot{x}(t)=f\left(x(t), u_{k}, d_{k}\right), x\left(t_{k}\right)=x_{k}\right\}^{\text {The Lagrangian of }(10) \text { is }}$

$$
\mathcal{L}(p, y, z)=\phi(p)-y^{\prime} b(p)-z^{\prime} c(p)
$$




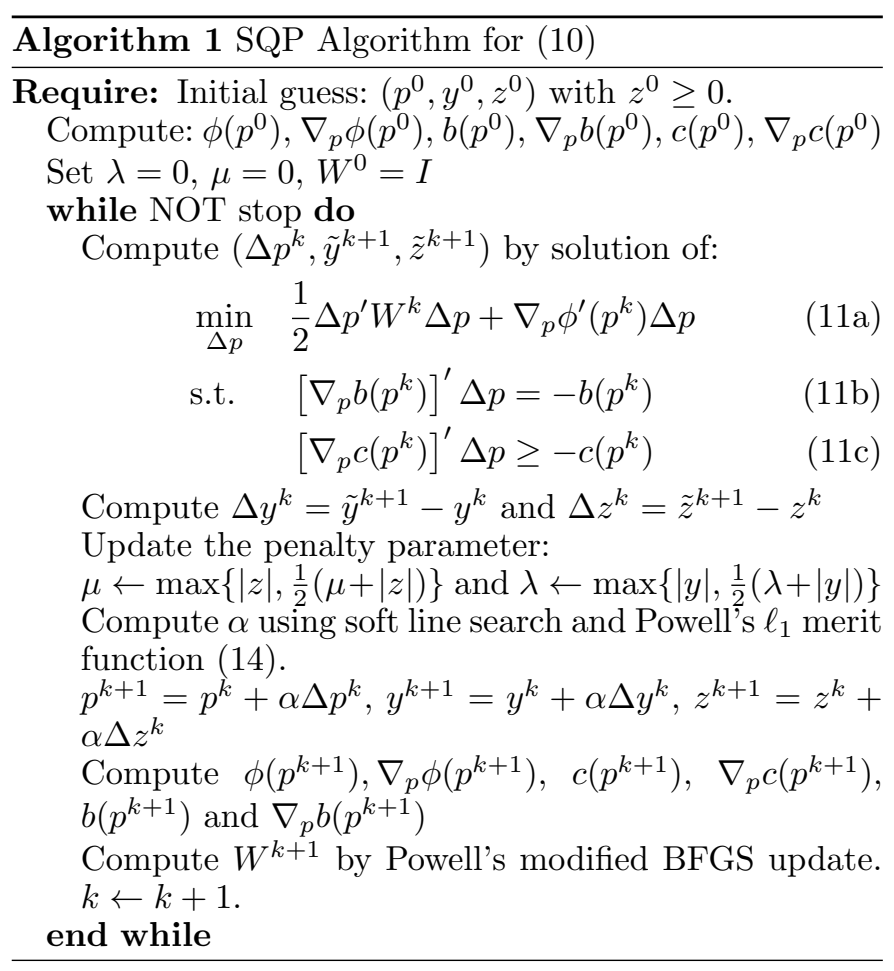

and the first order KKT conditions

$$
\begin{aligned}
& \nabla_{p} \mathcal{L}(p, y, z)=\nabla_{p} \phi(p)-\nabla_{p} b(p) y-\nabla_{p} c(p) z=0 \\
& b(p)=0 \\
& c(p) \geq 0 \\
& z \geq 0 \\
& c_{i}(p)=0 \vee z_{i}=0 \quad \forall i
\end{aligned}
$$

are used to test convergence of the SQP algorithm (Alg. $1)$.

The steps for solution of (10) by an SQP algorithm with line search are listed in Algorithm 1. The line search is based on Powell's $\ell_{1}$ penalty function

$$
P(p)=\phi(p)+\lambda^{\prime}|b(p)|+\mu^{\prime}|\min \{0, c(p)\}|
$$

and the Armijo sufficient decrease condition. The penalty vectors, $\lambda$ and $\mu$, are selected such that they are numerically larger than the corresponding Lagrange multipliers, i.e. $\lambda \geq|y|$ and $\mu \geq z$ where $y$ is the Lagrange multipliers associated with (10b) and $z$ is the Lagrange multipliers associated with (10c).

\subsection{Gradient Computation}

The most time consuming computations in Algorithm 1 are computation of the objective function $\phi(p)$, computation of the derivatives of the objective function $\nabla_{p} \phi(p)$, computation of the dynamics $b(p)$, and computation of the sensitivities, $\nabla_{p} b(p)$, associated with the dynamics. $b(p)$ and $\phi(p)$ are computed by evaluation of (4) and (5), respectively. Consequently

$$
\begin{aligned}
& \qquad b_{k}=b_{k}\left(x_{k}, x_{k+1}, u_{k}, d_{k}\right)=F_{k}\left(x_{k}, u_{k}, d_{k}\right)-x_{k+1} \\
& \nabla_{x_{k}} b_{k}=\nabla_{x_{k}} F_{k}\left(x_{k}, u_{k}, d_{k}\right)=S_{x_{k}}\left(t_{k+1}\right)^{\prime}=A_{k}^{\prime} \\
& \nabla_{u_{k}} b_{k}=\nabla_{u_{k}} F_{k}\left(x_{k}, u_{k}, d_{k}\right)=S_{u_{k}}\left(t_{k+1}\right)^{\prime}=B_{k}^{\prime} \\
& \nabla_{x_{k+1}} b_{k}=-I \\
& \text { where } x\left(t_{k+1}\right)=F\left(x_{k}, u_{k}, d_{k}\right) \text { and }
\end{aligned}
$$

$$
\begin{aligned}
\dot{x}(t) & =f\left(x(t), u_{k}, d_{k}\right) \\
\dot{S}_{x_{k}}(t) & =\left(\frac{\partial f}{\partial x}\left(x(t), u_{k}, d_{k}\right)\right) S_{x_{k}}(t) \\
\dot{S}_{u_{k}}(t) & =\left(\frac{\partial f}{\partial x}\left(x(t), u_{k}, d_{k}\right)\right) S_{u_{k}}(t)+\left(\frac{\partial f}{\partial u}\left(x(t), u_{k}, d_{k}\right)\right)
\end{aligned}
$$

with the initial conditions $x\left(t_{k}\right)=x_{k}, S_{x_{k}}\left(t_{k}\right)=I$, and $S_{u_{k}}\left(t_{k}\right)=0$. The stage cost and the associated derivatives are computed as

$$
\begin{aligned}
& G_{k}=G_{k}\left(x_{k}, u_{k}, d_{k}\right)= \int_{t_{k}}^{t_{k+1}} g\left(x(t), u_{k}, d_{k}\right) d t \\
& q_{k}=\nabla_{x_{k}} G_{k}=\int_{t_{k}}^{t_{k+1}}\left(\frac{\partial g}{\partial x}\left(x(t), u_{k}, d_{k}\right)\right) S_{x_{k}}(t) d t \\
& r_{k}=\nabla_{u_{k}} G_{k}=\int_{t_{k}}^{t_{k+1}} {\left[\left(\frac{\partial g}{\partial x}\left(x(t), u_{k}, d_{k}\right)\right) S_{u_{k}}(t)\right.} \\
&\left.+\left(\frac{\partial g}{\partial u}\left(x(t), u_{k}, d_{k}\right)\right)\right] d t
\end{aligned}
$$

The derivatives $\nabla_{x_{k}} b_{k}$ and $\nabla_{x_{k}} G_{k}$ are computed for $\left\{x_{k}\right\}_{k=1}^{N-1}$ and $k \in \mathcal{N}$. These derivatives are not computed for $x_{0}$ as $x_{0} \notin p$, i.e. $x_{0}$ is a fixed parameter of the optimisation problem but not a decision variable. The derivatives $\nabla_{u_{k}} b_{k}$ and $\nabla_{u_{k}} G_{k}$ are computed for $k \in \mathcal{N}$. The derivatives with respect to $x_{N}$ are

$$
\begin{aligned}
\nabla_{x_{N}} b_{N-1} & =-I \\
p_{N} & =\nabla_{x_{N}} \phi=\nabla_{x_{N}} h\left(x_{N}\right)
\end{aligned}
$$

In evaluation of the functions and derivatives needed in the SQP algorithm, i.e. evaluation of $\phi(p), \nabla_{p} \phi(p), b(p)$, and $\nabla_{p} b(p)$, the major computational task is solution to the differential equations (16) and evaluation of the associated quadrature equations (17). The Hovorka model is a nonstiff system of differential equations. Therefore, we use an embedded Dormand-Prince explicit Runge-Kutta scheme (DOPRI54) described in Dormand and Prince (1980) for solution of the differential equations (16) and integration of the quadrature equations (17). The Butcher tableau (see Butcher (2003)) of the DOPRI54 method is listed in Table 1. The DOPRI54 method has 7 stages, the advancing step, $x$, has order 5 , and the error estimator, $e$, has order 4 . A special DOPRI54 method tailored for solution of (16)(17) has been implemented. In this implementation, we re-use the internal stages computed by solution of (16) in the evaluation of the quadrature equation (17). The implementation uses an adaptive time step based on PIcontrol developed by Gustafsson (1992).

When $p$ is given as in the multiple shooting algorithm, evaluation of $c(p)$ and $\nabla_{p} c(p)$ becomes trivial. As $c(p)$ represents the bound constraints, $u_{\min } \leq u_{k} \leq u_{\max }$ for $k \in \mathcal{N}, \nabla_{p} c(p)$ is a constant and the corresponding constraints in the quadratic program (11) become bound constraints as well.

\section{APPLICATION TO AN ARTIFICIAL PANCREAS}

In this section we state and discuss the objective function and the scenarios used in the simulations. We also state the strategy for the non-linear model predictive controller. 
Table 1. Butcher tableau for the DOPRI54 method.

\begin{tabular}{c|ccccccc}
0 & 0 & 0 & 0 & 0 & 0 & 0 & 0 \\
$\frac{1}{5}$ & $\frac{1}{5}$ & 0 & 0 & 0 & 0 & 0 & 0 \\
$\frac{3}{10}$ & $\frac{3}{40}$ & $\frac{9}{40}$ & 0 & 0 & 0 & 0 & 0 \\
$\frac{4}{5}$ & $\frac{44}{55}$ & $\frac{-56}{15}$ & $\frac{32}{9}$ & 0 & 0 & 0 & 0 \\
$\frac{8}{9}$ & $\frac{19372}{6561}$ & $\frac{-25360}{2187}$ & $\frac{64448}{6561}$ & $\frac{-212}{729}$ & 0 & 0 & 0 \\
1 & $\frac{9017}{3168}$ & $\frac{-355}{33}$ & $\frac{46732}{5247}$ & $\frac{49}{176}$ & $\frac{-5103}{18656}$ & 0 & 0 \\
1 & $\frac{35}{384}$ & 0 & $\frac{500}{1113}$ & $\frac{125}{192}$ & $\frac{-2187}{6784}$ & $\frac{11}{84}$ & 0 \\
\hline$x$ & $\frac{5179}{57600}$ & 0 & $\frac{7571}{16695}$ & $\frac{393}{640}$ & $\frac{-92097}{339200}$ & $\frac{187}{2100}$ & $\frac{1}{40}$ \\
$\hat{x}$ & $\frac{35}{384}$ & 0 & $\frac{500}{1113}$ & $\frac{125}{192}$ & $\frac{-2187}{6784}$ & $\frac{11}{84}$ & 0 \\
\hline$e$ & $\frac{71}{57600}$ & 0 & $\frac{-71}{16695}$ & $\frac{71}{1920}$ & $\frac{-17253}{339200}$ & $\frac{22}{525}$ & $\frac{-1}{40}$
\end{tabular}

\subsection{Non-linear Model Predictive Control (NMPC)}

NMPC is a receding horizon control technology that repeatedly solves open-loop non-linear optimal control problems and implements the computed optimal control associated to the current time period (see e.g. Rawlings and Mayne (2009)). In this contribution, we use a receding horizon strategy to compute the ideal insulin administration profile for people with type 1 diabetes. In order to obtain the ideal insulin profile, the NMPC uses state feedback and relative long prediction horizons.

\subsection{Objective Function with Soft Output Constraints}

The objective of the insulin administration is to compensate glucose excursions caused by meals and variations in endogenous glucose production and utilisation. We use a penalty function defined as

$$
\begin{aligned}
\rho(G(t))=\frac{\kappa_{1}}{2} & |\max \{0, G(t)-\bar{G}\}|^{2} \\
& +\frac{\kappa_{2}}{2}|\max \{0, \bar{G}-G(t)\}|^{2} \\
& +\frac{\kappa_{3}}{2}\left|\max \left\{0, G(t)-G_{U}\right\}\right|^{2} \\
& +\frac{\kappa_{4}}{2}\left|\max \left\{0, G_{L}-G(t)\right\}\right|^{2}
\end{aligned}
$$

$G(t)$ is the blood glucose concentration, $\bar{G}=5 \mathrm{mmol} / \mathrm{L}$ is the target value for the blood glucose concentration, $G_{L}=4 \mathrm{mmol} / \mathrm{L}$ is a lower acceptable limit on the glucose concentration, and $G_{U}=8 \mathrm{mmol} / \mathrm{L}$ is an upper acceptable limit on the blood glucose concentration. The weights $\kappa_{1}-\kappa_{4}$ are used to balance the desirability of different deviations from the target. As hypoglycaemia is considered worse than hyperglycaemia, $\kappa_{1}<\kappa_{2}$ and $\kappa_{3}<\kappa_{4}$. The penalty function used in the simulations is illustrated in Fig. 4. $G(t)$ is a function of the state, $x(t)$, in the Hovorka model. Therefore, the penalty function (19) may be expressed as a stage cost in the form $g(x(t), u(t))$. The objective function used in the simulations is

$$
\phi=\int_{t_{0}}^{t_{f}} g(x(t), u(t)) d t+\frac{\eta}{2} \sum_{k=0}^{N-1}\left\|\Delta u_{k}\right\|_{2}^{2}
$$

where $\Delta u_{k}=u_{k}-u_{k-1}$. This objective function has no cost-to-go function, i.e. $h\left(x\left(t_{f}\right)\right)=0$, and can be brought into the standard form (3a) using state augmentation formulated by Rawlings and Mayne (2009).

We use $u_{\min }=0$ and a large $u_{\max }$ such that the upper bound is never active. We do the optimisation in a 24

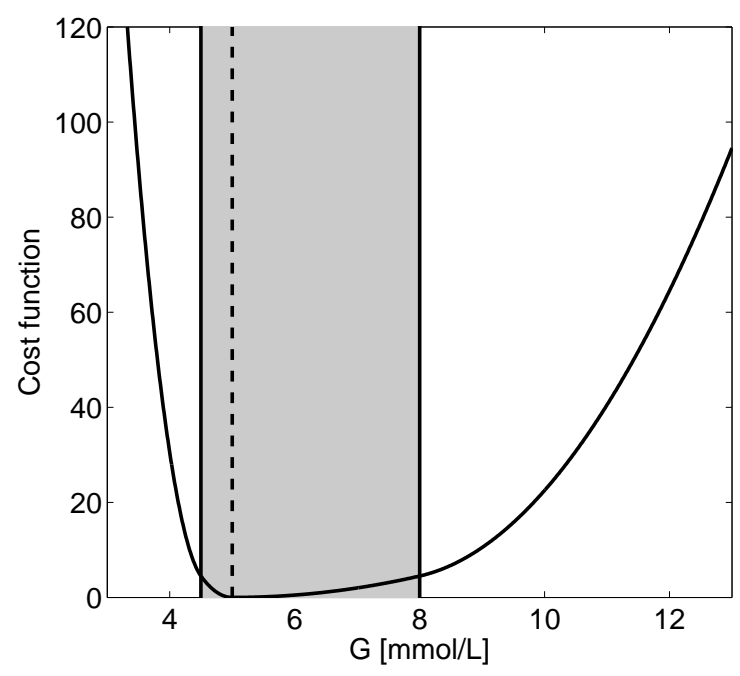

Fig. 4. Penalty as a function of the blood glucose concentration. The green shaded area is an interval of acceptable glucose concentrations. The target glucose concentration is $5 \mathrm{mmol} / \mathrm{L}$. Blood glucose concentrations less than $3 \mathrm{mmol} / \mathrm{L}$ is very undesirable as people may fall into coma at this low blood glucose concentration.

hour window, i.e. $t_{0}=0 \mathrm{~min}$ and $t_{f}=24 \cdot 60 \mathrm{~min}$, using a sampling time of $T_{s}=5 \mathrm{~min}$. In the scenario considered, the simulated $70 \mathrm{~kg}$ subject has a $62 \mathrm{~g} \mathrm{CHO}$ meal at 6:00, a $55 \mathrm{~g}$ CHO meal at 12:00, and a $50 \mathrm{~g} \mathrm{CHO}$ meal at 18:00. To ensure an optimal blood glucose profile, a prediction horizon of six hours, i.e., $N=6 \cdot 12=72$ samples, is employed in the receding horizon strategy.

\section{SIMULATION RESULTS}

In this Section, we use the model developed by Hovorka et al. (2004) and the developed multiple shooting SQP algorithm for (1) to compute insulin administration profiles for people with type 1 diabetes.

Fig. 5 illustrates an optimal insulin administration profile for the described scenario in the case where the controller knows the size and time of all meals in advance. Knowing the meal times and sizes allows the controller to deliver anticipatory insulin to pre-empt postprandial hyperglycaemia. However, the assumption that the patient would know in advance - and with accuracy - the meal times and sizes is not practical. Safety considerations would preclude significant amounts of insulin from being delivered prior to mealtime (as in this ideal scenario).

Fig. 6 shows the simulation results for the case in which the meals are announced to the MPC only at mealtime. Thus, the controller can deliver no anticipatory insulin prior to meals. The limitations for this case force the subject into (mild) hyperglycaemia, but hypoglycaemia is avoided. The insulin delivery profile for this case looks quite similar to bolus delivery of insulin by a pen; most of the meal-related insulin is delivered in bolus form in the few samples after the meals are taken (and announced). Simulated optimal bolus treatment with a pen provides glucose profiles comparable to the glucose profile in Fig. 6 . 

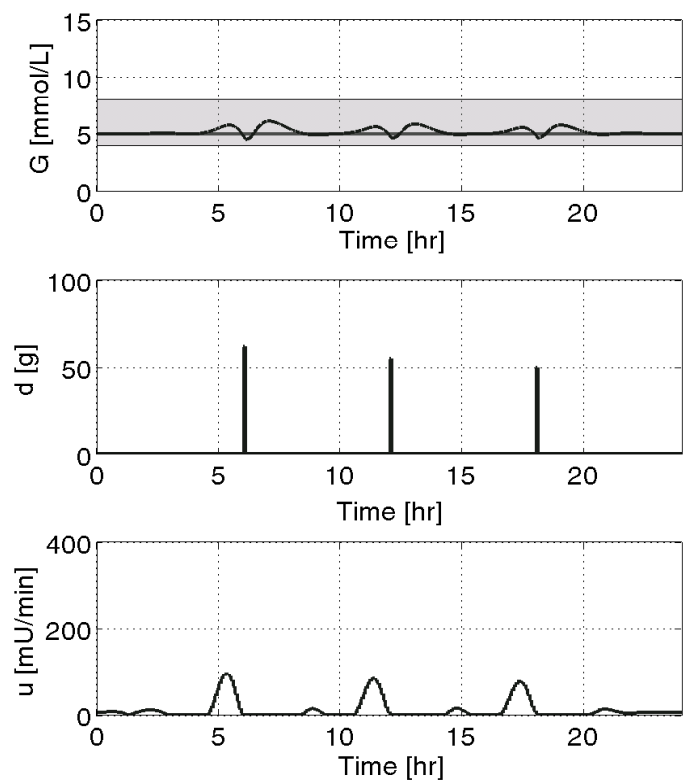

Fig. 5. Glucose profile (top), meal disturbances (middle) and optimal insulin administration profile (bottom) for the case with meal announcement in advance of the meal. Most insulin is taken before the meals.
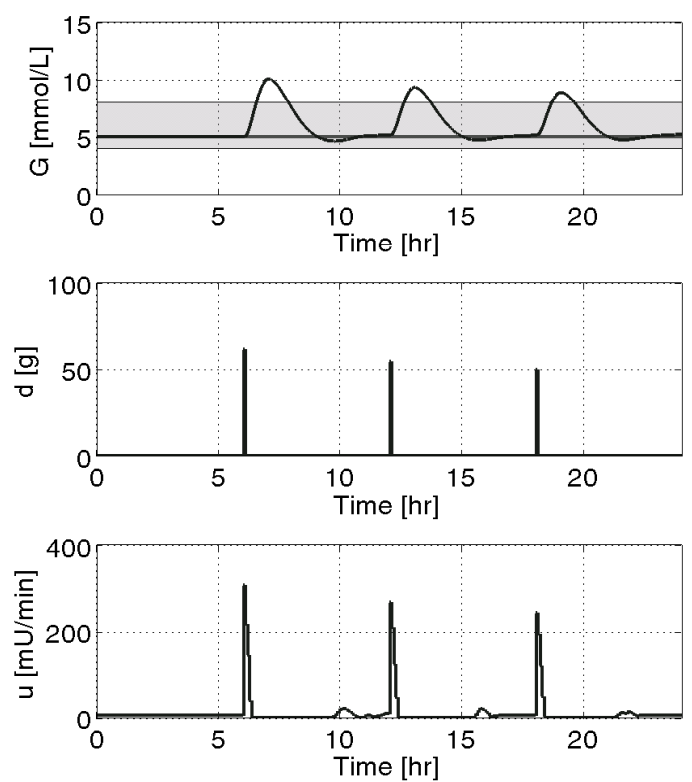

Fig. 6. Glucose profile (top), meal disturbances (middle) and optimal insulin administration profile (bottom) with meal announcement at meal time. Most insulin is taken in bolus like form at meal time.

These results demonstrate that for realistic cases, i.e., cases for which meal information is unknown until mealtime, reasonably good control can still be obtained. Perhaps more importantly, the bolus like nature of the insulin profile in this case suggests that a pen-based system may be able to achieve control comparable to that of a pump.

\section{CONCLUSION}

In this paper, we described a multiple shooting SQP algorithm for solution of a bound constrained continuoustime Bolza problem. Based on the model developed by Hovorka et al. (2004) for people with type 1 diabetes, we use our optimal control algorithm to compute insulin administration profiles for the cases with and without meal announcement in advance. The insulin profile for the realistic case with announcement of meals at mealtime is bolus like. This suggests that insulin treatment based on pen-systems may be nearly as effective as insulin treatment based on pump systems.

\section{REFERENCES}

Bock, H. and Plitt, K. (1984). A multiple shooting method for direct solution of optimal control problems. 242247. Proc. of the IFAC 9th World Congress, Budapest, Hungary.

Butcher, J.C. (2003). Numerical Methods for Ordinary Differential Equations. Wiley, Chichester, England.

Diehl, M., Ferreau, J., and Haverbeke, N. (2009). Efficient numerical methods for nonlinear MPC and moving horizon estimation. In L. Magni, D.M. Raimondo, and F. Allgöwer (eds.), Nonlinear Model Predictive Control. Towards New Challenging Applications, 391-417. Springer, Berlin, Germany.

Dormand, J.R. and Prince, P.J. (1980). A family of embedded runge-kutta formulae. Journal of Computational and Applied Mathematics, 6(1), 19 - 26.

Gustafsson, K. (1992). Control of Error and Convergence in ODE Solvers. Ph.D. thesis, Department of Automatic Control, Lund Institute of Technology.

Hovorka, R., Canonico, V., Chassin, L.J., Haueter, U., Massi-Benedetti, M., Federici, M.O., Pieber, T.R., Schaller, H.C., Schaupp, L., Vering, T., and Wilinska, M.E. (2004). Nonlinear model predictive control of glucose concentration in subjects with type 1 diabetes. Physiological Measurement, 25, 905-920.

Jørgensen, J.B. (2005). Moving Horizon Estimation and Control. Ph.D. thesis, Department of Chemical Engineering, Technical University of Denmark.

Klonoff, D.C., Cobelli, C., Kovatchev, B., and Zisser, H.C. (2009). Progress in development of an artificial pancreas. Journal of Diabetes Science and Technology, 3, 1002-1004.

Rao, C.V., Wright, S., and Rawlings, J.B. (1998). Application of interior-point methods to model predictive control. Journal of Optimization Theory and Applications, 99(3), $723-757$.

Rawlings, J.B. and Mayne, D.Q. (2009). Model Predictive Control: Theory and Design. Nob Hill Publishing, Madison, Wisconsin, USA.

World Health Organization (2009). Diabetes (fact sheet no. 312). WHO Web site:

http://www.who.int/mediacentre/factsheets/fs312/en/. 